\title{
INFLUENCE OF METASTABLE ARGON ATOMS AND DUST PARTICLES ON GAS DISCHARGE PLASMA
}

\author{
A.V. FEDOSEEV, ${ }^{1}$ G.I. SUKHININ ${ }^{1,2}$ \\ ${ }^{1}$ S. Kutateladze Institute of Thermophysics, \\ Siberian Branch of Russian Academy of Science \\ (1, Lavrentyeva Ave., Novosibirsk 630090, Russia; e-mail: fedoseev@itp. nsc. ru) \\ ${ }^{2}$ Novosibirsk State University \\ (2, Pirogova Str., Novosibirsk 630090, Russia)
}

The model of a DC glow discharge with metastable argon atoms and dust particles based on the Boltzmann equation for the electron energy distribution function (EEDF), dust particle charging, and balance equation for metastable argon atoms is presented. The processes of direct and stepwise electron impact ionization, metastable-metastable collisions, and recombination of electrons and ions on the dust particle surface and discharge tube wall are taken into account. The results show that the densities of metastable argon atoms and dust particles are in strong correlation, and both sufficiently influence gas discharge parameters.

\section{Introduction}

Dusty or complex plasma is an ionized gas of electrons, ions, and negatively charged micron-sized particles [13]. Dust grains can be found either in space (e.g., planet rings, interstellar molecular clouds, and cometary tails) or in different technological processes (e.g., plasma chemical deposition and coating, thermonuclear reactors, etc.) Under laboratory conditions, dusty plasmas are also intensively investigated in the positive column (PC) of a $\mathrm{DC}$ glow discharge and in $\mathrm{RF}$ discharges in noble gases at a low gas density. Many interesting phenomena are observed and investigated in dusty plasma, e.g., the formation of dusty structures (Coulomb crystals, liquids, and gases), phase transitions, vortices, wave propagation, and various kinetic processes. Laboratory dusty plasma in DC or RF discharges consists of electrons and ions with densities $n_{e} \sim n_{i} \sim 10^{7}-10^{9} \mathrm{~cm}^{-3}$, dust particles with dust number density $N_{d} \sim 10^{2}-10^{8} \mathrm{~cm}^{-3}$, and charge number $e Z_{d} \sim 10^{3}-10^{5} e$. For a small Havnes parameter, $P_{\mathrm{H}}=Z_{d} N_{d} / n_{e} \ll 1$, the charge of dust particles is determined only by plasma conditions. With increase in $P_{\mathrm{H}}$, the local parameters in the plasma region that contains dust particles (electron density and EEDF) change, which leads, in turn, to a change of the average charge of dust particles and, hence, of all properties of dusty plasma.

The influence of dust particles on discharge properties for the conditions of RF discharge used for the preparation of thin films in the semiconductor industry was investigated in 1992 with the help of particle-in-cell Monte Carlo simulations by J.P. Boeuf [4]. Recently, the dusty plasma of RF discharge was investigated by I. Denysenko et al. [5] with the help of the Boltzmann equation for EEDF. Various models for the reactive dusty plasma of $\mathrm{RF}$ discharge were also presented by W.J. Goedheer et al. [6] and I.V. Schweigert et al. [7]. The self-consistent model based on the Boltzmann equation for EEDF, OML (orbital motion limited) model for dust particle charging, with regard for the condition of quasineutrality was presented in [8]. It is understood that each dust particle acts as an electron and ion sink, and a large concentration of dust particles would have some effect on the plasma properties and on the plasma sustainment conditions. The electron and ion losses on dust particles should be compensated in ionizing collisions, and the averaged electric field in a discharge should increase in the region containing dust particles. With increase in the electric field in a dusty cloud, the double layer is forming, i.e. one side of the dusty cloud is positively charged, and another side is negatively charged. Such a cloud should be polarized in the presence of an external electric field. Different aspects of the influence of metastable atoms in DC [9] and RF [10] discharges, as well as in electron beam plasma [11], were presented. In works [12-14], the results of experimental investigations of metastable argon atoms and dust particles in gas discharges are reported. It is shown that the metastable argon atoms and dusty particles strongly influence the discharge plasma. Depending on discharge parameters, the presence of dust particles has both positive and negative effects on the metastable density [12]. The increase of the metastable density up to ten times was detected 
in the discharge with dusty particles in comparison with pristine argon plasma [14].

\section{Model}

We present a model that describes the influence of metastable argon atoms and dust particles on gas discharge parameters. The numerical model is based on a previously developed kinetic model of a low pressure DC glow discharge with dust particles based on the Boltzmann equation for EEDF [8] and the balance equation for metastable argon atoms. The model considers the ionization balance, the formation of EEDF, dust particle charging, and balance for metastable argon atoms. The homogeneous stationary Boltzmann equation for the isotropic part of EEDF $f_{0}$ in the presence of dusty particles and metastable argon atoms can be written in the form

$$
\begin{aligned}
& -\frac{\left(e_{0} E\right)^{2}}{3} \frac{\partial}{\partial U}\left(\frac{U}{H^{\prime}(U)} \frac{\partial f_{0}}{\partial U}\right)= \\
& =S_{\mathrm{el}}\left(f_{0}\right)+\sum_{k} S_{k}^{\mathrm{in}}\left(f_{0}\right)+S_{\text {ion }}\left(f_{0}\right)+S_{\text {stpw }}\left(f_{0}\right)+ \\
& +S_{p}\left(f_{0}\right)+S_{w}\left(f_{0}\right)+S_{d}\left(f_{0}\right)
\end{aligned}
$$

where $H^{\prime}(U)=N_{g}\left(\sum_{k} Q_{k}^{\text {in }}(U)+Q^{\mathrm{el}}(U)\right)+N_{d} \pi r_{0}^{2}(1-$ $\left.\left|e \varphi_{d}\right| / U\right), \varphi_{d}$ is the dust particle potential, $N_{d}$ is the density of dust particles with radius $r_{0}, N_{g}$ is the neutral argon density, $Q^{\mathrm{el}}(U)$ is the transport cross-section of scattering in elastic collisions, $Q_{k}^{\text {in }}(U)$ is the cross-section of the $k$-th inelastic collision, $U$ is the electron kinetic energy, $S_{\text {el }}$ and $S_{k}^{\text {in }}$ are the integrals of elastic and $k$-th inelastic collisions, and $E$ is the electric field strength. The ionization balance is determined by the processes of direct electron impact ionization $S_{\text {ion }}$, stepwise ionization from metastable argon states $S_{\text {stpw }}$, ionization in metastable-metastable collisions $S_{p}$, and recombination of electrons and ions on the dust particle surface $S_{d}$ and on the discharge tube wall $S_{w}$. The following balance equation for metastable density $N_{m}$ is used:

$$
\begin{aligned}
& \frac{\partial N_{m}}{\partial t}=\nu_{\text {exc }, m} N_{g}+k_{\text {rec }} N_{e} N_{i}-D_{m} \frac{N_{m}}{R^{2}}- \\
& -k_{m m} N_{m}^{2}-\nu_{\text {stpw }} N_{m}-\pi r_{0}^{2} N_{m} N_{d}\left\langle v_{m}\right\rangle- \\
& -k_{2 B} N_{g} N_{m}-k_{3 B} N_{g}^{2} N_{m} .
\end{aligned}
$$

Here, the excitation frequency of metastables $\nu_{\mathrm{exc}, m}$ and the ionization frequency from metastable atoms $\nu_{\text {stpw }}$ are calculated from the Boltzmann equation, $D_{m}$ is the metastable diffusion coefficient, $R$ is the radius of the discharge tube wall, $k_{m m}$ is the rate constant of metastablemetastable collisions, $N_{e}, N_{i}$ are the densities of electrons and ions, $\left\langle v_{m}\right\rangle$ is the mean velocity of metastables, $k_{\text {rec }}, k_{2 B}$, and $k_{3 B}$ are the rate constants of electron-ion radiative recombination and two- and three-body recombination, respectively.

The condition of quasineutrality in the dusty cloud is as follows:

$N_{i}=N_{e}+N_{d} Z_{d}$

The dust particle charge $Z_{d}$ is determined within the OML model. The quasineutrality condition, the Boltzmann equation (1) for EEDF, and the metastable balance equation (2) are solved numerically in a selfconsistent way for various dust particles concentrations $N_{d}$. For the convergence of the iterative procedure, the condition of ionization balance is used. The rates of production of argon metastable states in the electron impact excitation processes, $\nu_{\text {exc, } m}\left(f_{0}\right)$, and losses in the processes of stepwise ionization, $\nu_{\text {stpw }}\left(f_{0}\right)$, are calculated with the help of the Boltzmann equation. In the Boltzmann equation (1), the additional sources of electrons produced in the metastable-metastable collisions and in the stepwise ionization are taken from the calculation of Eq. (2). A possible emission of electrons from the surface of dust particles after the absorption of metastables is also considered.

\section{Results}

In Fig. 1, the self-consistent solutions for the dependences of the dust particle potential, $\varphi_{s}$, and the axial electric field, $E_{z}$, on the dust particle concentration, $N_{d}$, are presented for dust particle radii $r_{0}=1,2$, and $5 \mu \mathrm{m}$ without regard for the metastable argon atoms. It is seen that the self-consistent electric field increases sharply with the dust particle concentration in the region $N_{d} r_{0}^{2}>10^{-2} \mathrm{~cm}^{-1}$ due to increased electron losses in the process of recombination on dust particles. There is a smaller decrease of the particle potential with increase in the concentration of dust particles. It becomes only two times smaller, when the product of the dust particle concentration and the radius achieves the value $N_{d} r_{0} \sim 10^{2} \mathrm{~cm}^{-2}$. It is of interest that, for relatively small dust particle concentrations and for various dust particle radii $r_{0}$, the electric field is a function of the parameter $N_{d} r_{0}^{2}$. Moreover, in almost the whole $N_{d}$-region, 


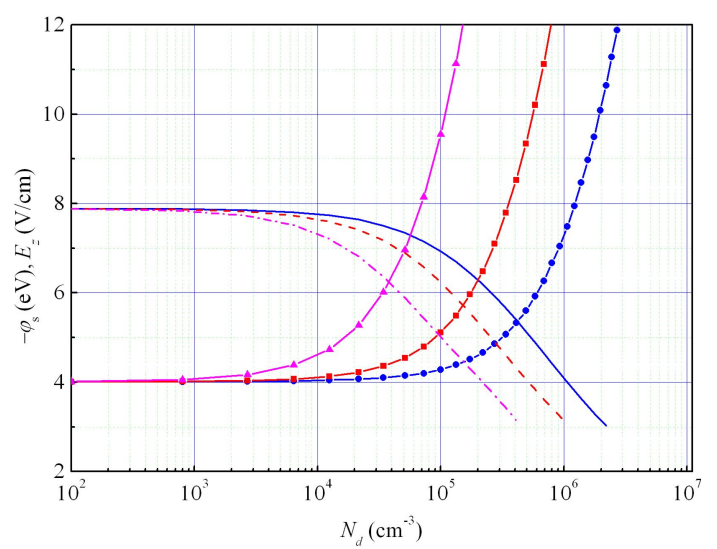

Fig. 1. Dust particle surface potential (lines), $\varphi_{s}$, and axial electric field (symbols), $E_{z}$, versus the dust particle concentration, $N_{d}$, for various dust particle radii: solid line and circles for $r_{0}=1 \mu \mathrm{m}$, dashed line and squares for $r_{0}=2 \mu \mathrm{m}$ and dash-dotted line and triangles for $r_{0}=5 \mu \mathrm{m}$. $N_{g}=1.75 \times 10^{16} \mathrm{~cm}^{-3}, j_{z}=1 \mathrm{~mA} / \mathrm{cm}^{2}$. Helium

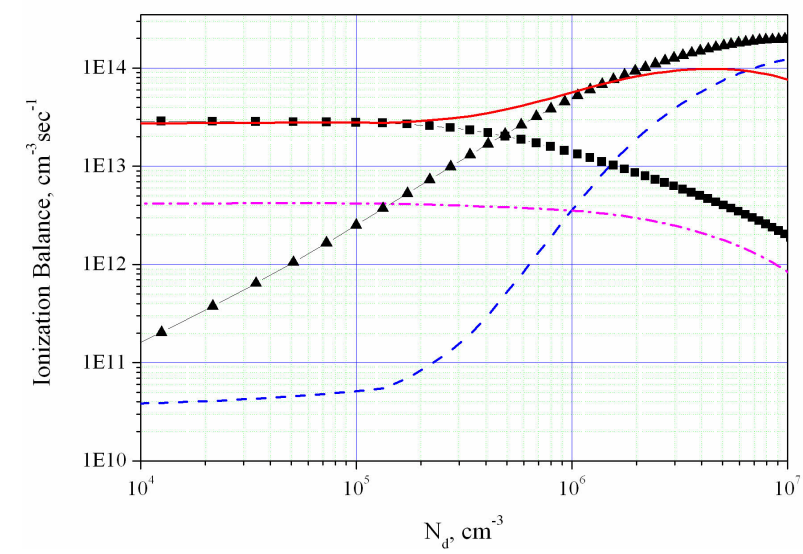

Fig. 2. The ionization balance terms: $S_{p}$ - solid line, $S_{\text {ion }}-$ dashed line, $S_{\text {stpw }}$ - dash-dotted line, $S_{d}-$ triangles, and $S_{w}-$ squares

the dust particle potential is a function of the parameter $N_{d} r_{0}$.

The terms of the Boltzmann equation (1) (see Fig. 2) and the metastable balance equation (2) (see Fig. 3) are presented for the following parameters of gas discharge: the electron current density $j_{e}=0.1 \mathrm{~mA} / \mathrm{cm}^{2}$, initial electric field $E_{0}=2 \mathrm{~V} / \mathrm{cm}$, argon pressure $p=0.5$ Torr, $r_{0}=10^{-4} \mathrm{~cm}$.

For the present conditions and for a small dust particle density, the ionization rate in metastable-metastable collisions exceeds the direct electron impact ionization rate. With increase in the dust particle density, the direct electron ionization becomes more important, and the electron recombination on the dust particle surface plays a great role. Metastable argon states are produced mainly

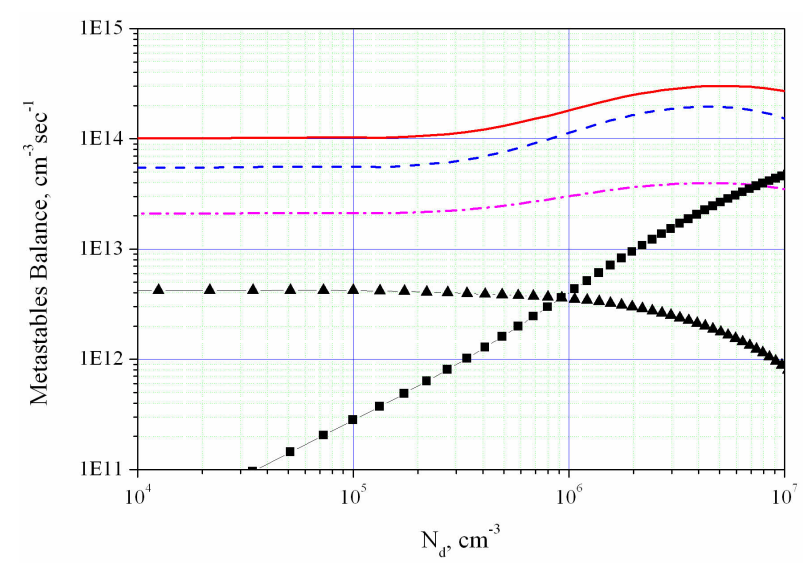

Fig. 3. The metastable balance terms: $\nu_{\text {exc }, m} N_{g}-$ solid line, $k_{m m} N_{m}^{2}$ - dashed line, $D_{m} N_{m} / R^{2}$ - dash-dotted line, $\nu_{\text {stpw }} N_{m}$ - triangles, and $r_{0}^{2} N_{m} N_{d}\left\langle\nu_{m}\right\rangle$ - squares

in the course of excitation by an electron impact from argon ground states including cascading from upper levels. The metastable-metastable collisions, the diffusion of metastables to the wall, and the diffusion of metastables to the dust particle surface at large densities of dust particles $N_{d}$ are the main processes of losses of metastables. The results show that the metastable argon atoms strongly influence the ionization balance. The density of metastable argon atoms depends to a great extent on the parameters of gas discharge and dust particles. Depending on conditions, the addition of dust particles into the discharge can lead to either an increase or a decrease of the density of metastable argon atoms. The results show that, for small dust particle density $N_{d}$, the ionization rate in metastable-metastable collisions can exceed the direct electron impact ionization rate.

\section{Conclusion}

It should be stressed that, even without dusty particles, the glow discharge in cylindrical tubes is a very complex open non-equilibrium system of neutral atoms (in various electronic states), ions, and electrons. For some conditions, the low-pressure glow discharge can be selforganized (stratification of a positive column) with nonlocal processes playing an important role. The addition of dust particles increases the complexity of the description of a glow discharge. The presence of metastable argon states strongly influences both the electron energy distribution function and the dust particle charging. Here, we consider a simplified 1D model that can highlight, nevertheless, the main problems of the inter- 
ference of dust particles, metastable argon states, and plasma parameters of a discharge.

As we deal with a multiparameter problem, it should be considered carefully, and all the rates and coefficients must be specified more precisely. Depending on conditions, the addition of dust particles into the discharge can lead to either an increase or a decrease of the density of metastable argon atoms $N_{m}$. In a wide range of gas discharge parameters (the gas pressure varies from 1 to $1000 \mathrm{~Pa}$, and the discharge current varies from $10^{-1}$ to $10^{1} \mathrm{~mA}$ ), the various processes can play a major role or can be even neglected. We note that the dust particles in a DC glow discharge are mainly situated at the center of the discharge tube, by disturbing the ionization balance there. Hence, the non-local problem for the axial and radial distributions of all plasma and dust particles and the parameters of a DC discharge should be considered.

The work was supported in part by Federal Targeted program Research and Educational Staff in Innovation Russia (contract no. 02.740.11.0109).

1. P.K. Shukla, Phys. of Plasmas 8, 1791 (2001).

2. V.E. Fortov et al., Phys. Usp. 47, 447 (2004).

3. O. Ishihara, J. Phys. D: Appl. Phys. 40, R121-R147 (2007).

4. J.P. Boeuf, Phys. Rev. A 46, 7910 (1992).

5. I. Denysenko, M.Y. Yu, K. Ostrikov, and A. Smolyakov, Phys. Rev. E 70, 046403 (2004).

6. W.J. Goedheer, M.R. Akdim, and Yu.I. Chutov, Contrib. Plasm. Phys. 44, 395 (2004).

7. I.V. Schweigert, A.L. Alexandrov, D.A. Ariskin et al., Phys. Rev. E 78, 026410 (2008).

8. G.I. Sukhinin and A.V. Fedoseev, Phys. Rev. E 81, $016402(2010)$.
9. A. Bogaerts and R. Gijbels, Phys. Rev. A 52, 3743 (1995).

10. M. Roberto, H.B. Smith, and J.P. Verboncoeur, IEEE Trans. Plas. Sci. 31, 1292 (2003).

11. G.I. Sukhinin, A.V. Fedoseev, and S.Ya. Khmel', Plasma Phys. Reports 34, 60 (2008).

12. H.T. Do, V. Sushkov, and R. Hippler, New J. of Phys. 11, 033020 (2009).

13. S. Mitic, M.Y. Pustylnik, abd G.E. Morfill, New J. of Phys. 11, 083020 (2009).

14. I. Stefanovic, N. Sadeghi, and J. Winter, in 19th International Symposium on Plasma Chemistry, edited by A. von Keudell, J. Winter, M. Böke, V. Schulz-von der Gathen (Univ. of Bochum, Bochum, 2009).

Received 19.01.11

ВПЛИВ МЕТАСТАБІЛЬНИХ АТОМІВ АРГОНУ

І ПИЛОВИХ ЧАСТИНОК НА ПЛАЗМУ

ГАЗОВОГО РОЗРЯДУ

А.В. Федосеєв, Г.І. Сухінін

Р е $з$ ю м е

У роботі подано модель тліючого розряду постійної напруги з метастабільними атомами аргону і пиловими частинками на основі обчислення рівняння Больцмана для функції розподілу електронів за енергією, моделі зарядки пилових частинок i рівняння балансу метастабільних атомів аргону. Враховано процеси прямої і ступінчастої іонізації електронним ударом, зіткнення метастабільних атомів між собою, рекомбінації електронів та іонів на поверхні пилової частинки і на поверхні розрядної трубки. Результати показали, що густини метастабільних атомів аргону і пилових частинок знаходяться у строгій залежності один від одного і суттєво впливають на параметри газового розряду. 